We present a case of primary mediastinal germinoma in a female. Germ cell tumours occur most commonly in the gonads but may occasionally arise in other areas, including the mediastinum. A mediastinal germinoma is an identical tumour to a gonadal seminoma, and are thought to originate from misplaced primordial germ cells retained in extra-gonadal sites. The affected individuals are overwhelmingly male with less than 10 cases in females reported within the English literature. It is important that the presence of a primary tumour within the ovary is excluded before the tumour is accepted as a primary mediastinal lesion. We highlight the current immunohistochemical diagnostic contribution and the need for accurate diagnosis in this rare presentation.

\section{JUXTAGLOMERULAR CELL TUMOUR IN A 29 YEAR OLD MALE}

Michael Stapelberg, Mary-Ann Koh, Gordon Wright

Pathology Queensland, Gold Coast University Hospital, Gold Coast, Queensland, Australia

Juxtaglomerular cell tumour is a rare renal neoplasm arising from the juxtaglomerular apparatus. Functioning and non-functioning tumours have been described. Functioning tumours are associated with excess renin production and clinical sequelae such as secondary hypertension and hyperaldosteronism. ${ }^{1,3}$ The majority of cases of juxtaglomerular cell tumour reported so far, occur in young adults and are benign. Only very few malignant cases have been reported. ${ }^{2}$ We report a case of a 29 year old male, with a well-defined solitary lesion within the left kidney which was initially biopsied and found to have the morphological and immunohistochemical features in keeping with a rennin secreting juxtaglomerular cell tumour. The patient underwent laparoscopic nephrectomy of the left kidney. We review some of the recent cases and discuss the common clinical presentations and the histological findings including immunohistochemistry and ancillary tests.

\section{References}

1. Martin SA, Mynderse LA, Lager DJ, et al. Juxtaglomerular cell tumor: a clinicopathologic study of four cases and review of the literature. Am J Clin Pathol 2001; 116: 854-63.

2. Duan X, Bruneval P, Hammadeh R, et al. Metastatic juxtaglomerular cell tumor in a 52 year old man. Am J Surg Pathol 2004; 28: 1098-102.

3. Kuroda N, Gotoda H, Ohe C, et al. Review of juxtaglomerular cell tumor with focus on pathobiological aspect. Diagn Pathol 2011; 26: 6:80.

\section{RADIOTHERAPY-INDUCED MALIGNANT PERIPHERAL NERVE SHEATH TUMOUR - A CASE REPORT}

Yi Sun, Md Firoz Iqbal

Nepean Hospital, Department of Anatomical Pathology, Australia

Malignant peripheral nerve sheath tumours (MPNST) are rare soft tissue tumours known to arise in a background of neurofibromatosis type 1 syndrome (NF1), previous radiation exposure or sporadically.
We report a case of a MPNST arising in a 66-year-old man who received radiotherapy localised to the neck 20 years prior for Hodgkin lymphoma. He has no personal or familial history of NF1 and is currently in remission for lymphoma.

His symptoms comprised progressive dysarthria, dysphagia, tongue deviation and weight loss. Examination demonstrated right hypoglossal nerve palsy and a right neck mass.

Imaging showed a FDG-avid, well-demarcated solid ovoid mass in the right carotid space.

FNA of the mass showed cohesive clusters of pleomorphic spindle cells with stripped nuclei and prominent nucleoli, accompanied by occasional bizarre giant cells. Stains for keratin, SMA, desmin, CD34, LCA were negative. Core biopsy showed isolated S-100 positive atypical spindle cells. The provisional diagnosis of MPNST was made and excisional biopsy suggested. Tumour resection with right neck dissection and parotidectomy was performed. Intraoperatively, the tumour appeared adjacent to the right accessory nerve and extended into the jugular foramen.

Macroscopically, the $60 \mathrm{~mm}$ smooth rubbery mass showed a pale tan solid cut surface with patchy haemorrhage, with a segment of the right accessory nerve associated with the capsule.

Microscopically, the well-circumscribed tumour comprised a diffuse fascicular proliferation of spindle cells with central necrosis. The cells had fibrillary cytoplasmic processes, elongated undulating pleomorphic and hyperchromatic nuclei, accompanied by abundant atypical mitoses. The loose and myxoid stroma contained congested hyalinised blood vessels, mast cells and extravasated blood. The spindle cells showed strong diffuse S100 positive nuclear staining. The edge of the tumour was contiguous with the adjacent nerve.

No evidence of local recurrence or distant metastasis was identified at the time of resection or at 1-year follow-up.

\section{EFFECT OF MELITTIN TREATMENT IN A MOUSE MODEL OF XENOBIOTIC-INDUCED CHOLANGITIS AND BILIARY FIBROSIS}

\author{
$\underline{\text { Hyun-Jung Sung }}^{1,2}$, Kyung-Hyun Kim ${ }^{1}$, Young-In Maeng ${ }^{1}$, \\ Kwan-Kyu Park \\ ${ }^{1}$ Department of Pathology, Catholic University of Daegu School \\ of Medicine, Daegu, and ${ }^{2}$ Department of Pathology, Sunlin \\ Hospital, Pohang, Republic of Korea
}

Background: Cholangiopathy is a chronic immune-mediated disease of the liver, which is characterized by cholangitis, ductular reaction and biliary type of hepatic fibrosis. There is no proven medical therapy that change the course of the disease. In previous studies, melittin is known for attenuation of hepatic injury, inflammation and hepatic fibrosis. We herein investigate whether melittin provide inhibition on cholangitis and biliary fibrosis in vivo.

Methods: 3,5-Diethoxycarbonyl-1,-dihydrocollidine (DDC) feeding mice is well-established model to study for cholangitis and biliary fibrosis. To investigate the effects of melittin on cholangiopathy, mice fed with $0.1 \%$ DDC diet with or without melittin treatment for 4 weeks. Liver morphology, serum markers of liver injury and cholestasis markers for inflammation of liver, degree of ductular reaction and degree of liver fibrosis were compared between with or without melittin treated DDC fed mice. 